\title{
Impact of non-hepatic hyperammonemia on mortality in intensive care unit patients: a retrospective cohort study
}

Jae Heon Kim ${ }^{1,2,}{ }^{\star}$, Hankyu Jeon ${ }^{1,2,}$, Sang Soo Lee ${ }^{1,2,3}$, I Re Heo ${ }^{1,2}$, Jung Woo Choi ${ }^{1}$, Hee Jin Kim ${ }^{1,2,3}$, Ra Ri Cha ${ }^{1,2,3}$, Jae Min Lee $e^{1,2,3}$, and Hyun Jin Kim ${ }^{1,2,3}$

${ }^{1}$ Department of Internal Medicine, Gyeongsang National University Hospital, Jinju; ${ }^{2}$ Department of Internal Medicine, Gyeongsang National University Changwon Hospital, Changwon; ${ }^{3}$ nstitute of Health Sciences, Gyeongsang National University, Jinju, Korea

Received: January 11, 2021 Revised : March 18, 2021 Accepted: April 26, 2021

\section{Correspondence to}

Sang Soo Lee, M.D.

Department of Internal

Medicine, Gyeongsang National University Changwon Hospital, 11 Samjeongja-ro, Seongsan-gu, Changwon 51472, Korea

Tel: +82-55-214-3711

Fax: +82-55-214-1036

E-mail:3939lee@naver.com https://orcid.org/0000-00031029-3436

*These authors contributed equally to this work.
Background/Aims: The effect of hyperammonemia on the mortality in patients with liver cirrhosis is well documented. However, little is known about the impact of hyperammonemia on mortality among intensive care unit patients without hepatic disease. We aimed to investigate factors associated with non-hepatic hyperammonemia among intensive care unit patients and to evaluate the factors related to the 7- and 90-day mortality.

Methods: Between February 2016 and February 2020, 948 patients without hepatic disease who had 972 episodes of admission to the intensive care unit were retrospectively enrolled and classified as hyperammonemia grades $\mathrm{o}(\leq 8 \mathrm{o} \mu \mathrm{g} / \mathrm{dL} ; 585$ [60.2\%]), 1 ( $\leq 160 \mu \mathrm{g} / \mathrm{dL} ; 291$ [29.9\%]), 2 ( $\leq 240 \mu \mathrm{g} / \mathrm{dL} ; 55$ [5.7\%]), and 3 (> 240 $\mu \mathrm{g} / \mathrm{dL} ; 41$ [4.2\%]). Factors associated with hyperammonemia and the 7- and 90-day mortality were evaluated by multivariate logistic regression analysis and Cox regression analysis, respectively. Kaplan-Meier survival curves for the 7- and 90-day mortality were constructed.

Results: The independent risk factors for hyperammonemia were male sex (odds ratio, 1.517), age (0.984/year), acute brain failure (2.467), acute kidney injury (1.437), prothrombin time-international normalized ratio (2.272/unit), and albumin (0.694/g/dL). The 90-day mortality rate in the entire cohort was $24.3 \%$ and gradually increased with increasing hyperammonemia grade at admission $(17.9 \%$, $28.2 \%, 43.6 \%$, and $61.0 \%$ in patients with grades $0,1,2$, and 3 , respectively). Additionally, non-hepatic hyperammonemia was an independent predictor of the $90-$ day mortality in intensive care unit patients.

Conclusions: Non-hepatic hyperammonemia is common (39.8\%) and associated with the 90 -day mortality among intensive care unit patients.

Keywords: Hyperammonemia; Non-hepatic disease; Intensive care units; Acute brain failure; Mortality

\section{INTRODUCTION}

Hyperammonemia is defined as a raised level of serum ammonia, a nitrogen-containing compound. In adults, this is associated with liver cirrhosis in $90 \%$ of cases [1]. Hepatic hyperammonemia may play a central role in the development of acute life-threatening encephalopathy that can lead to cerebral edema, brain stem herni- 
ation, and death [2]. In a recent study, serum ammonia level was correlated to encephalopathy severity and was an independent predictor of mortality in patients with liver cirrhosis [3]. Hepatic hyperammonemia has neurotoxic effects and causes inflammation and injury to several organs [4]. In general, hyperammonemia directly causes hepatic injury, immune system dysfunction, and hepatic stellate cell activation [5-8].

On the other hand, serum ammonia levels can be increased by non-hepatic factors. Hyperammonemia may occur from either increased ammonia production or decreased ammonia elimination. The etiology of increased ammonia production includes infection [9,10], hematologic malignancy [11,12], organ transplantation [13], and protein load and increased catabolism. Protein load and increased catabolism arise due to seizures, trauma, intensive exercise, starvation, steroid use, total parenteral nutrition (TPN), and gastrointestinal (GI) bleeding [14-17]. The etiology of decreased ammonia elimination includes portosystemic shunts [18], ureterosigmoidostomy [19], drugs (valproate and carbamazepine) [20,21], and inborn errors of metabolism [22].

Most patients with non-hepatic hyperammonemia present with confusion and coma $[23,24]$. Non-hepatic hyperammonemia among intensive care unit (ICU) patients is associated with an especially high risk of morbidity and mortality $[25,26]$. Although several reviews and case reports on hyperammonemia in patients with non-hepatic disease and ICU patients have been published, large observational studies on non-hepatic hyperammonemia are limited [24,26]. Prado et al [26]. reported that ammonia levels affect the mortality rates of ICU patients with non-hepatic disease and were correlated with mortality by univariate analysis using the chi-square test in 100 patients with non-hyperammonemia. Additionally, no significant factors associated with non-hepatic hyperammonemia were found in the Cox regression analysis.

Accordingly, our large, retrospective, observational study was designed to evaluate the characteristics of ICU patients with non-hepatic hyperammonemia. Our aim was to investigate factors associated with non-hepatic hyperammonemia among ICU patients and to elucidate whether ammonia levels affect mortality of ICU patients with non-hepatic diseases.

\section{METHODS}

\section{Study population}

This retrospective study included 4,235 consecutive episodes of ICU admission among 3,767 patients at Gyeongsang National University Changwon Hospital (GNUCH) from February 2016 to February 2020. The exclusion criteria were as follows: (1) patient ammonia levels were not measured ( $\mathrm{n}=2,973)$; (2) hyperbilirubinemia (> $2 \mathrm{mg}$ / $\mathrm{dL} ; \mathrm{n}=207) ;(3)$ liver cirrhosis $(\mathrm{n}=21)$; (4) hepatitis A, B, or $C$ virus infections $(n=9)$; (5) liver cancer $(n=6)$; (6) portosystemic shunt $(\mathrm{n}=2)$; and $(7)$ age $<18$ years $(\mathrm{n}=15)$. The remaining 972 episodes in 948 patients admitted to the ICU, whose ammonia levels were measured, were finally selected for analysis (Supplementary Fig. 1). All procedures performed in studies involving human participants were in accordance with the ethical standards of the Institutional Review Board of GNUCH (IRB File No. 2020-11-011). Informed consent was waived given that all of the personal data obtained were anonymized before analysis, as determined by the Institutional Review Boards of GNUCH. All methods in this study were performed in accordance with the ethical standards of the institutional research committee and with the 1964 Helsinki declaration.

\section{Data collection}

The patients' medical histories were reviewed to gather demographic data, including age; sex; body mass index; alcohol consumption; concomitant diseases, such as diabetes, underlying liver disease, congestive heart failure, extrahepatic malignancy, and end-stage renal disease; and laboratory data, including total bilirubin, white blood cell (WBC), hemoglobin, platelet count, blood urea nitrogen (BUN), creatinine, albumin, total cholesterol, aspartate aminotransferase, alanine aminotransferase, and prothrombin time-international normalized ratio (PT-INR). The main diagnostic categories at the time of admission to the ICU were classified as follows: sepsis, GI disorder, drug overdose, trauma, intracranial hemorrhage, neurologic disorder, seizure, cardiogenic disorder, respiratory disorder, and renal disorder.

Ammonia levels were measured with the Beckman Coulter AU 5800 clinical chemistry analyzer (Beckman Coulter, Brea, CA, USA). The normal blood ammonia level for an adult is $\leq 80 \mu \mathrm{g} / \mathrm{dL}$ using this device. There- 
fore, we defined hyperammonemia as an ammonia level $>80 \mu \mathrm{g} / \mathrm{dL}$. Additionally, we extracted information on potential risk factors for hyperammonemia: generalized seizures; hematologic malignancy; acute kidney injury (AKI); GI bleeding; gastric bypass surgery; TPN; infection; trauma; and intake of drugs, such as valproate, carbamazepine, proton pump inhibitor (PPI), and steroids.

\section{Follow-up and definition}

Medical charts were retrospectively reviewed to determine the 7- and 90-day mortality and non-hepatic hyperammonemia at admission. The index date was the date of first admission to the ICU. Hyperammonemia grades were defined as follows (serum ammonia concentrations in parentheses): (1) grade $O$ as patients without hyperammonemia $(\leq 80 \mu \mathrm{g} / \mathrm{dL})$; (2) grade 1 as ammonia levels 1 to 2 times the upper limit of normal (ULN) (81 to $160 \mu \mathrm{g} / \mathrm{dL}$ ); (3) grade 2 as ammonia levels 2 to 3 times the ULN ( 161 to $240 \mu \mathrm{g} / \mathrm{dL}$ ); and (4) grade 3 as ammonia levels $>3$ times the ULN ( $>240 \mu \mathrm{g} / \mathrm{dL}$ ). Further, AKI was defined as an absolute increase in serum creatinine by $\geq 0.3 \mathrm{mg} / \mathrm{dL}$ or $\geq 50 \%$ from baseline within 48 hours [27]. Acute brain failure was defined as a Glasgow Coma Scale (GCS) score $<15$ or a Full Outline of UnResponsiveness (FOUR) score $<16$ [28].

\section{Statistical analysis}

Continuous variables were expressed as medians (interquartile range). The Mann-Whitney $U$ test was performed to analyze quantitative data. The Fisher's exact and Pearson's chi-square tests were used for qualitative data. The factors associated with hyperammonemia were evaluated using a multivariate logistic regression model after adjusting for potential confounding variables. Risk analysis was performed by calculating the odds ratio (OR) and the 95\% confidence interval (CI). Kaplan-Meier survival curves for the 7- and 90-day mortality were constructed and compared using the log-rank test. Univariate and multivariate analyses were conducted using a Cox proportional regression model to identify potential factors associated with 7- and 90-day mortality. Risk was expressed as hazard ratios and 95\% CIs. A two-sided $p$ value $<0.05$ was considered statistically significant for all analyses. All statistical operations were performed using PASW Statistics version 18 (SPSS Inc., Chicago, IL, USA).

\section{RESULTS}

\section{Patient characteristics}

There was a total of 972 ICU admission among 948 patients who ammonia levels were measured (Table 1). Hyperammonemia episodes (387 [39.8\%]) were from significantly younger patients (median age, 66.o years) than the patients with the $585(60.2 \%)$ episodes without hyperammonemia (median age, 69.0 years; $p=0.003$ ). The proportion of men was higher among patients with (59.7\%) than without hyperammonemia (47.9\%, $p<0.001$ ). Additionally, the proportion of patients with endotracheal intubation and acute brain failure was higher among patients with than without hyperammonemia. However, there were no significant differences between patients with and without hyperammonemia in the rates of diabetes, alcohol consumption $>40 \mathrm{~g} /$ day, congestive heart failure, and end-stage renal disease. Laboratory findings revealed that patients with hyperammonemia had higher ammonia, WBC, BUN, creatinine, and PT-INR levels but lower albumin and total cholesterol levels than patients without hyperammonemia.

\section{Potential factors associated with hyperammonemia}

The rates of AKI, TPN, and infection were higher in patients with than in those without hyperammonemia (Supplementary Table 1). However, there were no significant differences between patients with and without hyperammonemia in the rates of generalized seizures, hematologic malignancy, GI bleeding, gastric bypass surgery, trauma, and drugs, such as valproate/carbamazepine, PPI, and steroids. Among the 10 principle diagnostic categories at the time to ICU admission, sepsis, seizure, and renal disorder were associated with a median ammonia level $\geq 80 \mu \mathrm{g} / \mathrm{dL}$ (Supplementary Table 2). Supplementary Fig. 2 shows the distribution of potential risk factors for the different hyperammonemia grades. Patients with AKI causing impaired urea excretion comprised $26.8 \%, 33.3 \%, 58.2 \%$, and $68.3 \%$ of patients with hyperammonemia grades $\mathrm{O}, 1,2$, and 3 , respectively. Patients with infection causing urease-producing organism comprised $36.1 \%, 43.3 \%, 52.7 \%$, and $52.1 \%$ of patients with hyperammonemia grades $0,1,2$, and 3 , respectively. The baseline characteristics according to the initial ammonia grades are reported in Supplementary Table 3. Most patients (66.4\%) in our cohort had acute brain 
Table 1. Baseline characteristics at admission according to the initial ammonia level

\begin{tabular}{|c|c|c|c|c|}
\hline Characteristic & Overall & Normal ammonia & High ammonia & $p$ value $^{a}$ \\
\hline Number & $972(100)$ & $585(60.2)$ & $387(39.8)$ & \\
\hline Age, yr & $68.0(55.0-77.8)$ & $69.0(57.0-78.0)$ & $66.0(54.0-76.0)$ & 0.003 \\
\hline Male sex & $511(52.6)$ & $280(47 \cdot 9)$ & $231(59 \cdot 7)$ & $<0.001$ \\
\hline $\mathrm{BMI}, \mathrm{kg} / \mathrm{m}^{2}$ & $23.1(20.6-25.6)$ & $23.1(20.7-25.5)$ & $23.1(20.2-25.7)$ & 0.790 \\
\hline Diabetes & $286(29.4)$ & $168(28.7)$ & $118(30.5)$ & 0.566 \\
\hline Alcohol > $40 \mathrm{~g} /$ day & $44(4 \cdot 5)$ & $24(4.1)$ & $20(5.2)$ & 0.435 \\
\hline $\mathrm{CHF}$ & $42(4 \cdot 3)$ & $24(4.1)$ & $18(4 \cdot 7)$ & 0.748 \\
\hline ESRD & $33(3.4)$ & $19(3.2)$ & $14(3 \cdot 6)$ & 0.857 \\
\hline Extrahepatic malignancy & $52(5 \cdot 3)$ & $28(4.8)$ & $24(6.2)$ & 0.383 \\
\hline Endotracheal intubation & $504(51.9)$ & $253(43.2)$ & $251(51.9)$ & $<0.001$ \\
\hline Acute brain failure & $645(66.4)$ & $343(58.6)$ & $302(78.0)$ & $<0.001$ \\
\hline Serum ammonia, $\mu \mathrm{g} / \mathrm{dL}$ & $72.0(54.0-101.0)$ & $58.0(46.0-68.0)$ & $111.0(92.0-160.0)$ & $<0.001$ \\
\hline WBC, $10 \times 10^{9} / \mathrm{L}$ & $12.0(7.9-16.5)$ & $11.3(7.8-15 \cdot 3)$ & $13.1(8.0-18.1)$ & 0.001 \\
\hline Hemoglobin, g/dL & $12.4(10.6-13.9)$ & $12.5(10.7-13.8)$ & $12.3(10.4-14.1)$ & 0.993 \\
\hline Platelet, $\times 10^{9} / \mathrm{L}$ & $222.0(168.0-275.7)$ & $222.0(172.0-269.0)$ & $223.0(160.0-286.0)$ & 0.753 \\
\hline BUN, mg/dL & $19.3(13.6-30.9)$ & $17.9(13.9-28.7)$ & $21.1(14.2-38.4)$ & $<0.001$ \\
\hline Creatinine, mg/dL & $0.93(0.65-1.48)$ & $0.86(0.65-1.33)$ & $1.03(0.67-1.80)$ & 0.001 \\
\hline Bilirubin, mg/dL & $0.68(0.50-0.93)$ & $0.68(0.51-0.91)$ & $0.68(0.48-1.00)$ & 0.962 \\
\hline Albumin, g/dL & $3.6(2.9-4.1)$ & $3.8(3.1-4.2)$ & $3.3(2.8-3.9)$ & $<0.001$ \\
\hline Total cholesterol, mg/dL & $153.0(116.0-189.0)$ & $159.0(123.0-196.5)$ & $145.0(112.0-179.0)$ & $<0.001$ \\
\hline PT-INR & $1.07(0.99-1.25)$ & $1.04(0.98-1.16)$ & $1.15(1.03-1.37)$ & $<0.001$ \\
\hline
\end{tabular}

Values are presented as number (\%) or median (interquartile range).

BMI, body mass index; CHF, congestive heart failure; ESRD, end-stage renal disease; WBC, white blood cell; BUN, blood urea nitrogen; PT-INR, prothrombin time-international normalized ratio.

${ }^{a}$ Mann-Whitney $U$ test and chi-square test.

failure, meaning decreased levels of consciousness. Serum ammonia levels were significantly and closely dependently associated with acute brain failure (Fig. 1A). Patients with acute brain failure comprised 58.6\%, 74.6\%, $85.5 \%$, and $92.7 \%$ of patients with hyperammonemia grades $\mathrm{O}, 1,2$, and 3 , respectively. At admission, albumin was inversely associated to ammonia levels, with WBC and PT-INR having a parallel relationship with ammonia levels (Supplementary Fig. 3).

Univariate analysis showed that male sex, age, acute brain failure, AKI, infection, WBC, PT-INR, total cholesterol, and albumin were related to hyperammonemia (Table 2). On multivariate analysis, the independent factors for hyperammonemia were male sex, age, acute brain failure, AKI, PT-INR, and albumin (Table 2).

\section{Impact of ammonia level on mortality}

The median ICU and hospital lengths of stay were 4.0 and 15.0 days, respectively. In our entire cohort, the 7and 90 -day mortality rate was $14.1 \%$ and $24.3 \%$, respectively. The median ammonia level in non-survivors was higher than in survivors $(87.0 \mu \mathrm{g} / \mathrm{dL}$ vs. $68.0 \mu \mathrm{g} / \mathrm{dL}, p<$ 0.001 ). A strong stepwise association was observed between hyperammonemia severity and 90-day mortality (Fig. 1B). Patients with grade o hyperammonemia (17.9\%) had a lower 90-day mortality than those with grades 1 (28.2\%, $p<0.001), 2$ (43.6\%, $p<0.001)$, and $3(61.0 \%, p<$ $0.001)$ (Fig. 2).

The factors associated with the 90-day mortality on univariate analysis were age, hyperammonemia grades 1 , 2, and 3 , acute brain failure, diabetes, extrahepatic malignancy, AKI, infection, platelet count, PT-INR, total cho- 

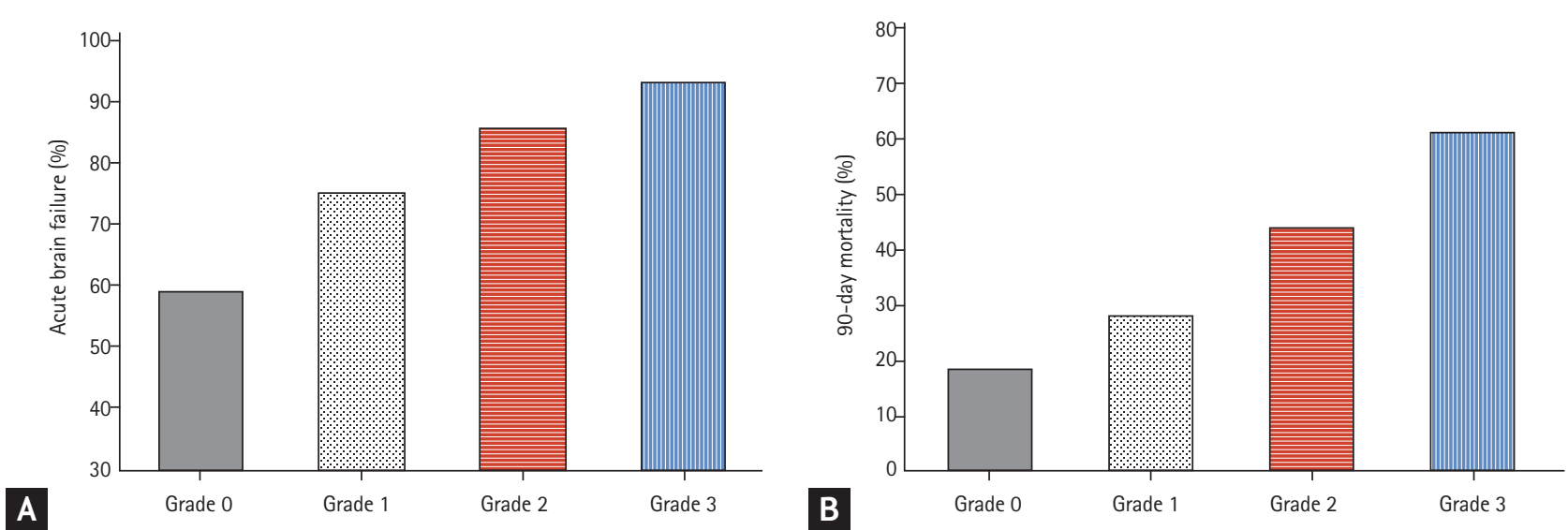

Figure 1. Rates of (A) acute brain failure and (B) 90-day mortality based on initial ammonia levels at admission ( $\mathrm{n}=972$ ).

Table 2. Univariate and multivariate analyses showing significant associated factors of hyperammonemia $(\mathrm{n}=972)$

\begin{tabular}{|c|c|c|c|c|}
\hline \multirow{2}{*}{ Variable } & \multicolumn{2}{|c|}{ Univariate analysis } & \multicolumn{2}{|c|}{ Multivariate analysis } \\
\hline & $p$ value & OR $(95 \% \mathrm{CI})$ & $p$ value & $\mathrm{OR}(95 \% \mathrm{CI})$ \\
\hline Male sex & $<0.001$ & $1.613(1.244-2.092)$ & 0.004 & $1.517(1.138-2.021)$ \\
\hline Age, yr & 0.006 & $0.988(0.980-0.997)$ & 0.001 & $0.984(0.974-0.993)$ \\
\hline Acute brain failure & $<0.001$ & $2.507(1.873-3.355)$ & $<0.001$ & $2.467(1.812-3.359)$ \\
\hline AKI & $<0.001$ & $1.861(1.416-2.445)$ & 0.027 & $1.437(1.041-1.982)$ \\
\hline Infection & 0.003 & $1.478(1.138-1.921)$ & 0.771 & $0.950(0.672-1.342)$ \\
\hline WBC, $10 \times 10^{9} / \mathrm{L}$ & 0.001 & $1.031(1.013-1.050)$ & 0.126 & $1.015(0.996-1.035)$ \\
\hline PT-INR & $<0.001$ & $3.350(2.156-5.207)$ & $<0.001$ & $2.272(1.473-3.506)$ \\
\hline Total cholesterol, mg/dL & $<0.001$ & $0.996(0.993-0.998)$ & 0.788 & $1.000(0.997-1.004)$ \\
\hline Albumin, g/dL & $<0.001$ & $0.642(0.544-0.757)$ & 0.002 & $0.694(0.549-0.878)$ \\
\hline
\end{tabular}

OR, odds ratio; CI, confidence interval; AKI, acute kidney injury; WBC, white blood cell; PT-INR, prothrombin time-international normalized ratio.

lesterol, and albumin (Table 3). The factors associated with 90-day mortality in the final multivariate analysis included age, hyperammonemia grades 1, 2, and 3, acute brain failure, platelet count, and albumin (Table 3). The same risk factors were identified for the 7-day mortality rate. The factors associated with 7-day mortality in the final multivariate analysis included age, hyperammonemia grades 2 and 3, acute brain failure, platelet, and albumin (Supplementary Table 4).

\section{DISCUSSION}

This large retrospective observational study included patients whose ammonia levels were measured in the ICU.
We found that the prevalence of non-hepatic hyperammonemia in the ICU was $39.8 \%$. The 90 -day mortality of patients with hyperammonemia was higher than that of those without hyperammonemia (33.9\% vs. $17.9 \%, p<$ 0.001). Additionally, the 90-day mortality rate was closely dependent and was affected by ammonia levels. This study showed that male sex, age, acute brain failure, AKI, PT-INR, and albumin are independent risk factors for the development of non-hepatic hyperammonemia. The occurrence of non-hepatic hyperammonemia in the ICU was an independent risk factor for 90-day mortality. In particular, non-hepatic hyperammonemia grade at admission correlated directly with the adjusted 90-day mortality, and hyperammonemia grades were independent predictors of the 9o-day mortality. 
Hyperammonemia results from increased ammonia production, decreased ammonia elimination, or both. Ammonia metabolism involves mainly five organs: liver, gut, kidney, muscle, and brain. Ammonia is produced primarily in the GI tract, but also in the kidneys

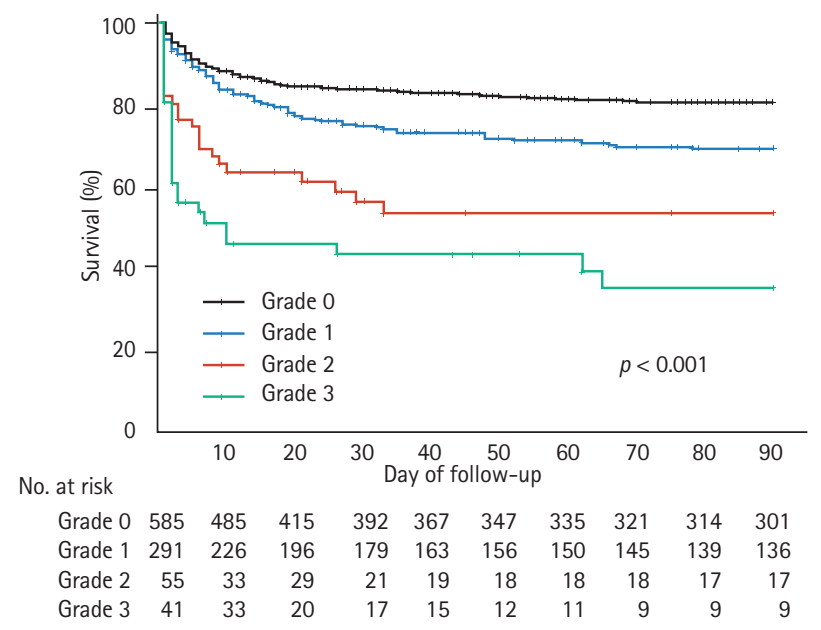

Figure 2. Kaplan-Meier survival curves for 90-day mortality according to initial ammonia levels $(n=972)$. and muscles [29]. Blood ammonia is metabolized to urea through the urea cycle in the liver. When the capacity of the liver to eliminate ammonia is exceeded ammonia metabolism is dependent on the kidney, muscles, and brain [30]. In the case of hyperammonemia, the kidney increases urinary excretion of ammonia. When the capacity of the kidney to metabolize ammonia is overwhelmed due to AKI, advanced hyperammonemia develops. Serum ammonia levels can be increased as a result of changes in acid-base status and GI bleeding [31]. In the case of increased muscle catabolism, through seizures, intense exercise, starvation, steroid use, or trauma, ammonia production increases. Moreover, TPN can lead to hyperammonemia by providing more protein to patients than usually consumed enterally [32]. It is well documented that drug-induced non-hepatic hyperammonemia is caused by valproate, carbamazepine, and PPI [33]. In our results, alcohol consumption $>40 \mathrm{~g} /$ day did not show a significant correlation with hyperammonemia. However, this may have bias due to the small number of patients with alcohol consumption > $40 \mathrm{~g} /$ day and limitations of retrospective studies.

Table 3. Univariate and multivariate analyses showing significant predictive factors of 90-days mortality $(\mathbf{n}=972)$

\begin{tabular}{|c|c|c|c|c|}
\hline \multirow{2}{*}{ Variable } & \multicolumn{2}{|c|}{ Univariate analysis } & \multicolumn{2}{|c|}{ Multivariate analysis } \\
\hline & $p$ value & $\operatorname{HR}(95 \% \mathrm{CI})$ & $p$ value & $\operatorname{HR}(95 \% \mathrm{CI})$ \\
\hline Age, yr & $<0.001$ & $1.025(1.015-1.035)$ & $<0.001$ & $1.025(1.014-1.035)$ \\
\hline \multicolumn{5}{|l|}{ Ammonia level $^{\mathrm{a}}$} \\
\hline Grade o & \multicolumn{2}{|c|}{ Reference } & & \\
\hline Grade 1 & 0.001 & $1.623(1.216-2.167)$ & 0.037 & $1.372(1.019-1.847)$ \\
\hline Grade 2 & $<0.001$ & $3.039(1.950-4.738)$ & 0.001 & $2.158(1.345-3.463)$ \\
\hline Grade 3 & $<0.001$ & $4.988(3.220-7.724)$ & $<0.001$ & $4.145(2.594-6.623)$ \\
\hline Acute brain failure & $<0.001$ & $3.461(2.406-4.978)$ & $<0.001$ & $3.026(2.085-4.390)$ \\
\hline Diabetes & 0.004 & $1.475(1.132-1.921)$ & 0.572 & $1.084(0.820-1.433)$ \\
\hline Extrahepatic malignancy & 0.006 & $1.859(1.198-2.882)$ & 0.157 & $1.392(0.880-2.201)$ \\
\hline AKI & $<0.001$ & $1.663(1.285-2.154)$ & 0.771 & $1.044(0.779-1.400)$ \\
\hline Infection & $<0.001$ & $2.087(1.614-2.699)$ & 0.087 & $1.302(0.963-1.762)$ \\
\hline Platelet, $\times 10^{9} / \mathrm{L}$ & 0.005 & $0.998(0.996-0.999)$ & 0.003 & $0.998(0.997-0.999)$ \\
\hline PT-INR, unit & 0.005 & $1.146(1.042-1.261)$ & 0.789 & 0.978 (0.829-1.153) \\
\hline Total cholesterol, mg/dL & $<0.001$ & $0.994(0.992-0.997)$ & 0.964 & $1.000(0.997-1.003)$ \\
\hline Albumin, g/dL & $<0.001$ & $0.559(0.484-0.647)$ & 0.001 & $0.714(0.582-0.876)$ \\
\hline
\end{tabular}

HR, hazard ratio; CI, confidence interval; AKI, acute kidney injury; PT-INR, prothrombin time-international normalized ratio.

${ }^{\mathrm{a}}$ Ammonia level: Grade $\mathrm{o} \leq 80 \mu \mathrm{g} / \mathrm{dL}, 80 \mu \mathrm{g} / \mathrm{dL}<$ Grade $1 \leq 160 \mu \mathrm{g} / \mathrm{dL}, 160 \mu \mathrm{g} / \mathrm{dL}<$ Grade $2 \leq 240 \mu \mathrm{g} / \mathrm{dL}$, and Grade $3>240 \mu \mathrm{g} / \mathrm{dL}$. 
Male sex, age, acute brain failure, AKI, PT-INR, and albumin were associated with non-hepatic hyperammonemia in ICU patients. These factors are thought to reflect protein load, increased catabolism, or decreased ammonia elimination. Ammonia balance is controlled by a fine equilibrium, which may be affected by age. A positive correlation between ammonia level and age was previously reported [34]. However, the present study found that the adjusted OR (0.984 per year) for hyperammonemia decreased with age. This phenomenon is difficult to explain clearly, but ammonia metabolism could have been affected in the critically ill patients comprising our cohort. Our results showed that hyperammonemia is more common in younger than older individuals (median 66.0 years vs. 69.0 years, respectively) and in males. The group with high ammonia showed higher median BUN level than the normal ammonia group. This finding possibly indicates that younger men with higher muscle mass are at higher risk for hyperammonemia, with higher BUN levels due to excessive muscle degradation within the context of an acute illness in the ICU. Acute brain failure, which entails a decreased level of consciousness, including cognitive impairment, abnormal neuropsychological test, and cerebral edema and herniation, reflects hyperammonemia. Because of the limitation of the retrospective nature of this study, we defined acute brain failure as a GCS score $<15$ or FOUR score $<16$ [28]. Patients with acute brain failure in our cohort consisted of patients with abnormal GCS (recorded at hospitalization in all ICU patients) or FOUR (mainly those undergoing endotracheal intubation or exhibiting abnormal respiration, confirmed through retrospective chart review) scores. A significant positive correlation was observed between grades of hyperammonemia and the rate of acute brain failure: prevalence of acute brain failure was 58.6\%, 74.6\%, 85.5\%, and $92.7 \%$ in patients with hyperammonemia grades $0,1,2$, and 3 , respectively. However, causes of acute brain failure do not only include hyperammonemia but also organic disorders such as intracranial hemorrhage and neurological disorders.

Few studies are available on the impact of non-hepatic hyperammonemia on mortality among ICU patients $[24,26]$. Sakusic et al. [24] found that serum ammonia levels in 167 ICU patients with non-hepatic hyperammonemia were not associated with increased mortality.
However, they did not compare patients with hyperammonemia to patients with normal ammonia levels [24]. Prado et al. [26] found that mortality rates among 100 ICU patients were significantly higher among those with mild and moderate non-hepatic hyperammonemia and those without non-hepatic hyperammonemia. However, they demonstrated a correlation only in univariate analysis because of the small number of patients. To the best of our knowledge, the present study demonstrated for the first time that non-hepatic hyperammonemia is an independent predictor of 90-day mortality using multivariate analysis in a large population of ICU patients. The 90-day mortality rate gradually increased with increasing hyperammonemia grades at admission. Additionally, we found approximately 0.3-, 1.2-, and 3.1fold increases in the risk of 90-day mortality in ICU patients with hyperammonemia grades 1, 2, and 3, respectively, than that in patients without hyperammonemia. In previous studies involving cirrhotic patients, serum ammonia levels correlated with the severity of hepatic encephalopathy and were an independent risk factor for mortality $[3,35]$. Our study showed that hyperammonemia is a significant predictor of mortality in ICU patients without hepatic disease.

Although the pathophysiology of non-hepatic hyperammonemia induced encephalopathy is poorly understood, hyperammonemia causes an increased blood ammonia level across the blood-brain-barrier and accumulation of extracellular glutamate in the brain. Acute ammonia intoxication causes activation of the N-methyl D-aspartate receptors and reversible and irreversible neurological disorders and the development of acute brain failure [36,37]. In our study, acute brain failure suggests encephalopathy, cerebral edema with astrocyte malfunction, and herniation, which are thought to have a significant effect on the high mortality rate.

The present study has a few limitations because of its retrospective nature. Ammonia metabolism is regulated by acid-base balance, but we were not able to determine the lactate level, an end product of anabolic metabolism. In addition, we could not measure the Sequential Organ Failure Assessment score or the Acute Physiology and Chronic Health Evaluation score due to limitations caused by the retrospective nature of this study. Of the 4,205 episodes of ICU admissions screened, 2,973 episodes were excluded as ammonia levels were not mea- 
sured at the time of admission. Of the 972 episodes of ICU admissions finally selected, we were unable to collect serial ammonia data after the initial ammonia level check. Therefore, we could not assess the response to hyperammonemia treatment on prognosis. Further, our results did not show arterial ammonia levels, which do not usually correlate with venous ammonia levels (venous ammonia levels vary locally) [38]. However, Ong et al. [35] found that venous and arterial ammonia levels were similar in their correlation with encephalopathy severity. Despite these limitations, the present study was conducted in a large population of ICU patients, and clearly showed a significant correlation between hyperammonemia grades and 90-day mortality.

In conclusion, male sex, younger age, acute brain failure, AKI, prolonged PT-INR, and decreased albumin levels were associated with hyperammonemia among ICU patients without hepatic disease. Our observations suggest that hyperammonemia occurs commonly among ICU patients without hepatic disease and has a significant effect on 9o-day mortality. Additionally, classification of hyperammonemia grades, as used in the present study, may help predict prognosis for ICU patients with non-hepatic disease.

\section{KEY MESSAGE}

1. The effect of hyperammonemia on the mortality in patients with liver cirrhosis is well documented, but little is known about the impact of hyperammonemia on mortality in intensive care unit (ICU) patients without hepatic disease.

2. Male sex, younger age, acute brain failure, acute kidney injury, prolonged prothrombin time-international normalized ratio, and decreased albumin levels were associated with non-hepatic hyperammonemia in ICU patients.

3. In addition, non-hepatic hyperammonemia is occurs commonly in ICU patients (39.8\%) and associated with 90 -day mortality.

\section{Conflict of interest}

No potential conflict of interest relevant to this article was reported.

\section{REFERENCES}

1. Laish I, Ben Ari Z. Noncirrhotic hyperammonaemic encephalopathy. Liver Int 2011;31:1259-1270.

2. Butterworth RF. Effects of hyperammonaemia on brain function. J Inherit Metab Dis 1998;21 Suppl 1:6-20.

3. Shalimar, Sheikh MF, Mookerjee RP, Agarwal B, Acharya SK, Jalan R. Prognostic role of ammonia in patients with cirrhosis. Hepatology 2019;70:982-994.

4. Tapper EB, Jiang ZG, Patwardhan VR. Refining the ammonia hypothesis: a physiology-driven approach to the treatment of hepatic encephalopathy. Mayo Clin Proc 2015;90:646-658.

5. Jia B, Yu ZJ, Duan ZF, et al. Hyperammonaemia induces hepatic injury with alteration of gene expression profiles. Liver Int 2014;34:748-758.

6. Albillos A, Lario M, Alvarez-Mon M. Cirrhosis-associated immune dysfunction: distinctive features and clinical relevance. J Hepatol 2014;61:1385-1396.

7. Shawcross DL, Wright GA, Stadlbauer V, et al. Ammonia impairs neutrophil phagocytic function in liver disease. Hepatology 2008;48:1202-1212.

8. Jalan R, De Chiara F, Balasubramaniyan V, et al. Ammonia produces pathological changes in human hepatic stellate cells and is a target for therapy of portal hypertension. J Hepatol 2016;64:823-833.

9. Cheang HK, Rangecroft L, Plant ND, Morris AA. Hyperammonaemia due to Klebsiella infection in a neuropathic bladder. Pediatr Nephrol 1998;12:658-659.

10. Samtoy B, DeBeukelaer MM. Ammonia encephalopathy secondary to urinary tract infection with Proteus mirabilis. Pediatrics 1980;65:294-297.

11. Kwan L, Wang C, Levitt L. Hyperammonemic encephalopathy in multiple myeloma. N Engl J Med 2002;346:1674-1675.

12. Lora-Tamayo J, Palom X, Sarra J, et al. Multiple myeloma and hyperammonemic encephalopathy: review of 27 cases. Clin Lymphoma Myeloma 2008;8:363-369.

13. Moffatt-Bruce SD, Pesavento T, Von Viger J, et al. Successful management of immunosuppression in a patient with severe hyperammonemia after lung transplantation. 
J Heart Lung Transplant 2008;27:801-803.

14. Graham TE. Exercise-induced hyperammonemia: skeletal muscle ammonia metabolism and the peripheral and central effects. Adv Exp Med Biol 1994;368:181-195.

15. Nakamura K, Yamane K, Shinohara K, et al. Hyperammonemia in idiopathic epileptic seizure. Am J Emerg Med 2013;31:1486-1489.

16. Seashore JH, Seashore MR, Riely C. Hyperammonemia during total parenteral nutrition in children. JPEN J Parenter Enteral Nutr 1982;6:114-118.

17. Olde Damink SW, Dejong CH, Jalan R. Review article: hyperammonaemic and catabolic consequences of upper gastrointestinal bleeding in cirrhosis. Aliment Pharmacol Ther 2009;29:801-810.

18. Elnekave E, Belenky E, Van der Veer L. Noncirrhotic extrahepatic portosystemic shunt causing adult-onset encephalopathy treated with endovascular closure. Case Rep Radiol 2015;2015:852853.

19. Cascino GD, Jensen JM, Nelson LA, Schutta HS. Periodic hyperammonemic encephalopathy associated with a ureterosigmoidostomy. Mayo Clin Proc 1989;64:653-656.

20. Gerstner T, Buesing D, Longin E, et al. Valproic acid induced encephalopathy: 19 new cases in Germany from 1994 to 2003: a side effect associated to VPA-therapy not only in young children. Seizure 2006;15:443-448.

21. Ambrosetto G, Riva R, Baruzzi A. Hyperammonemia in asterixis induced by carbamazepine: two case reports. Acta Neurol Scand 1984;69:186-189.

22. Batshaw ML, Brusilow SW. Treatment of hyperammonemic coma caused by inborn errors of urea synthesis. J Pediatr 1980;97:893-900.

23. Hawkes ND, Thomas GA, Jurewicz A, et al. Non-hepatic hyperammonaemia: an important, potentially reversible cause of encephalopathy. Postgrad Med J 2001;77:717-722.

24. Sakusic A, Sabov M, McCambridge AJ, et al. Features of adult hyperammonemia not due to liver failure in the ICU. Crit Care Med 2018;46:e897-e903.

25. Clay AS, Hainline BE. Hyperammonemia in the ICU. Chest 2007;132:1368-1378.

26. Prado FA, Delfino VD, Grion CM, de Oliveira JA. Hyperammonemia in ICU patients: a frequent finding associated with high mortality. J Hepatol 2015;62:1216-1218.
27. Khwaja A. KDIGO clinical practice guidelines for acute kidney injury. Nephron Clin Pract 2012;120:c179-c184.

28. Reddy DR, Singh TD, Guru PK, et al. Identification of acute brain failure using electronic medical records. J Crit Care 2016;34:12-16.

29. Bachmann C. Mechanisms of hyperammonemia. Clin Chem Lab Med 2002;40:653-662.

30. Olde Damink SW, Dejong CH, Deutz NE, et al. Kidney plays a major role in ammonia homeostasis after portasystemic shunting in patients with cirrhosis. Am J Physiol Gastrointest Liver Physiol 2006;291:G189-G194.

31. Olde Damink SW, Dejong CH, Deutz NE, van Berlo CL, Soeters PB. Upper gastrointestinal bleeding: an ammoniagenic and catabolic event due to the total absence of isoleucine in the haemoglobin molecule. Med Hypotheses 1999;52:515-519.

32. Lee JC, Lai HS, Huang SM, Chang CJ, Wang ST, Chen WJ. Hyperammonemic encephalopathy due to essential amino acid hyperalimentation. J Formos Med Assoc 1994;93:486-491.

33. Murphy JV, Marquardt K. Asymptomatic hyperammonemia in patients receiving $\mathrm{v}$ alproic acid. Arch Neurol 1982;39:591-592.

34. Maldonado C, Guevara N, Queijo C, Gonzalez R, Fagiolino P, Vazquez M. Carnitine and/or acetylcarnitine deficiency as a cause of higher levels of ammonia. Biomed Res Int 2016;2016:2920108.

35. Ong JP, Aggarwal A, Krieger D, et al. Correlation between ammonia levels and the severity of hepatic encephalopathy. Am J Med 2003;114:188-193.

36. Hermenegildo C, Monfort P, Felipo V. Activation of $\mathrm{N}$-methyl-D-aspartate receptors in rat brain in vivo following acute ammonia intoxication: characterization by in vivo brain microdialysis. Hepatology 2000;31:709-715.

37. Ahluwalia V, Betrapally NS, Hylemon PB, et al. Impaired gut-liver-brain axis in patients with cirrhosis. Sci Rep 2016;6:26800.

38. Clemmesen JO, Larsen FS, Kondrup J, Hansen BA, Ott P. Cerebral herniation in patients with acute liver failure is correlated with arterial ammonia concentration. Hepatology 1999;29:648-653. 
Supplementary Table 1. Potential factors associated with hyperammonemia at admission

\begin{tabular}{|c|c|c|c|c|}
\hline Variable & Overall & Normal ammonia & High ammonia & $p$ value \\
\hline Number & $972(100)$ & $585(60.2)$ & $387(39.8)$ & \\
\hline Generalized seizures & $33(3 \cdot 4)$ & $17(2.9)$ & $16(4.1)$ & 0.366 \\
\hline Hematologic malignancy & $6(0.6)$ & $2(0.3)$ & $4(1.0)$ & 0.223 \\
\hline AKI & $314(32.3)$ & $157(26.8)$ & $157(40.6)$ & $<0.001$ \\
\hline GI bleeding & $21(2.2)$ & $10(1.7)$ & $11(2.8)$ & 0.264 \\
\hline Gastric bypass surgery & $16(1.6)$ & $11(1.9)$ & $5(1.3)$ & 0.610 \\
\hline TPN & $6(0.6)$ & $1(0.2)$ & $5(1.3)$ & 0.040 \\
\hline Valproate/Carbamazepine & $4(0.4)$ & $2(0.3)$ & $2(0.5)$ & 0.652 \\
\hline PPI & $45(4.6)$ & $28(4.8)$ & $17(4 \cdot 4)$ & 0.876 \\
\hline Steroid & $23(2.4)$ & $14(2.4)$ & $9(2.3)$ & 1.000 \\
\hline Infection & $387(39.8)$ & $211(36.1)$ & $176(45 \cdot 5)$ & 0.004 \\
\hline Trauma & $58(6.0)$ & $38(6.5)$ & $20(5.2)$ & 0.411 \\
\hline
\end{tabular}

Values are presented as number (\%).

AKI, acute kidney injury; GI, gastrointestinal; TPN, total parenteral nutrition; PPI, proton pump inhibitor. 
Kim JH, et al. Non-hepatic hyperammonemia in ICU

Supplementary Table 2. Diagnostic categories at intensive care unit admission

\begin{tabular}{lcc}
\hline Diagnostic category & Events & Ammonia level, $\mu \mathrm{g} / \mathrm{dL}$ \\
\hline Sepsis & $129(13.3)$ & $80.0(56.5-134.5)$ \\
Gastrointestinal disorder & $54(5.6)$ & $68.0(47.5-100.5)$ \\
Drug overdose & $47(4.8)$ & $74.0(55.0-102.0)$ \\
Trauma & $58(6.0)$ & $64.0(55.8-83.0)$ \\
Intracranial hemorrhage & $144(14.8)$ & $65.0(50.3-83.0)$ \\
Neurologic disorder & $100(10.3)$ & $65.0(48.0-85.5)$ \\
Seizure & $19(2.0)$ & $85.0(61.0-117.0)$ \\
Cardiogenic disorder & $229(23.6)$ & $73.0(55.0-96.0)$ \\
Respiratory disorder & $154(15.8)$ & $79.5(58.0-113.3)$ \\
Renal disorder & $38(3.9)$ & $89.5(61.3-128.0)$ \\
\hline
\end{tabular}

Values are presented as number (\%) or median (interquartile range). 


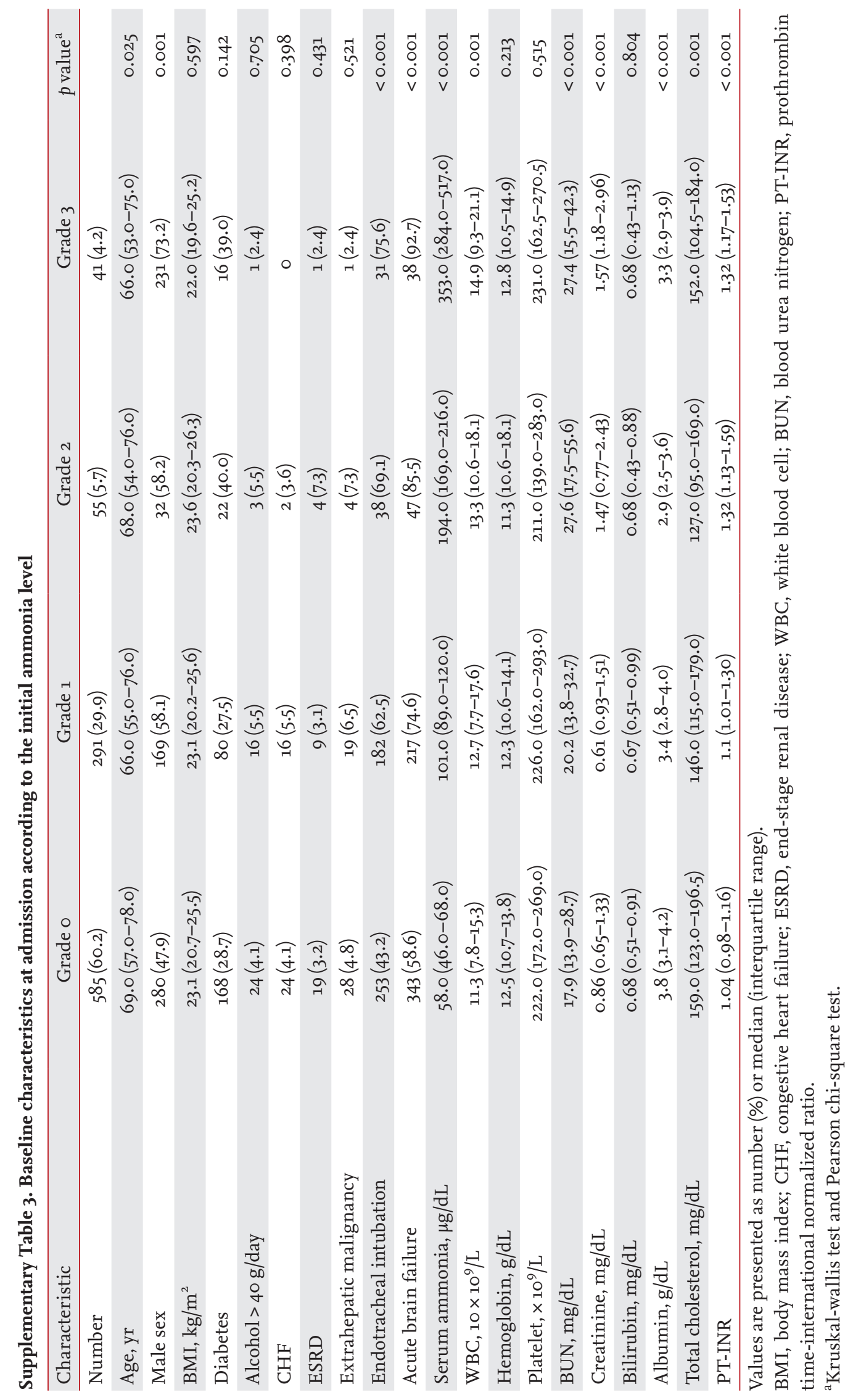


Supplementary Table 4. Univariate and multivariate analyses showing significant predictive factors of 7 -day mortality $(\mathbf{n}=\mathbf{9 7 2})$

\begin{tabular}{|c|c|c|c|c|}
\hline \multirow[t]{2}{*}{ Variable } & \multicolumn{2}{|c|}{ Univariate analysis } & \multicolumn{2}{|c|}{ Multivariate analysis } \\
\hline & $p$ value & $\operatorname{HR}(95 \% \mathrm{CI})$ & $p$ value & $\mathrm{HR}(95 \% \mathrm{CI})$ \\
\hline Male sex & 0.039 & $0.702(0.501-0.983)$ & 0.140 & $0.767(0.538-1.091)$ \\
\hline Age, yr & $<0.001$ & $1.026(1.013-1.040)$ & 0.001 & $1.024(1.009-1.038)$ \\
\hline \multicolumn{5}{|l|}{ Ammonia level $^{\mathrm{a}}$} \\
\hline Grade o & \multicolumn{2}{|c|}{ Reference } & & \\
\hline Grade 1 & 0.288 & $1.245(0.831-1.864)$ & 0.775 & $1.063(0.700-1.613)$ \\
\hline Grade 2 & $<0.001$ & $3.256(1.904-5.570)$ & $<0.001$ & $2.264(1.271-4.033)$ \\
\hline Grade 3 & $<0.001$ & $5.844(3.526-9.687)$ & $<0.001$ & $4.649(2.679-8.066)$ \\
\hline Acute brain failure & $<0.001$ & $3.349(2.063-5.436)$ & $<0.001$ & $2.903(1.761-4.786)$ \\
\hline Diabetes & 0.004 & $1.645(1.169-2.314)$ & 0.490 & $1.136(0.791-1.630)$ \\
\hline AKI & $<0.001$ & $1.920(1.372-2.686)$ & 0.277 & $1.239(0.842-1.825)$ \\
\hline Infection & 0.001 & $1.736(1.242-2.428)$ & 0.816 & $1.047(0.709-1.546)$ \\
\hline Platelet, $\times 10^{9} / \mathrm{L}$ & $<0.001$ & $0.996(0.994-0.998)$ & 0.001 & $0.997(0.995-0.999)$ \\
\hline PT-INR, unit & 0.006 & $1.175(1.048-1.318)$ & 0.909 & $0.988(0.807-1.210)$ \\
\hline Total cholesterol, mg/dL & 0.005 & $0.995(0.992-0.999)$ & 0.355 & $1.002(0.998-1.005)$ \\
\hline Albumin, g/dL & $<0.001$ & $0.551(0.455-0.667)$ & 0.001 & $0.641(0.495-0.831)$ \\
\hline
\end{tabular}

HR, hazard ratio; CI, confidence interval; AKI, acute kidney injury; PT-INR, prothrombin time-international normalized ratio.

${ }^{\mathrm{a}}$ Ammonia level: Grade $\mathrm{s} \leq 80 \mu \mathrm{g} / \mathrm{dL}, 80 \mu \mathrm{g} / \mathrm{dL}<$ Grade $1 \leq 160 \mu \mathrm{g} / \mathrm{dL}, 160 \mu \mathrm{g} / \mathrm{dL}<$ Grade $2 \leq 240 \mu \mathrm{g} / \mathrm{dL}$, and Grade $3>240 \mu \mathrm{g} / \mathrm{dL}$. 


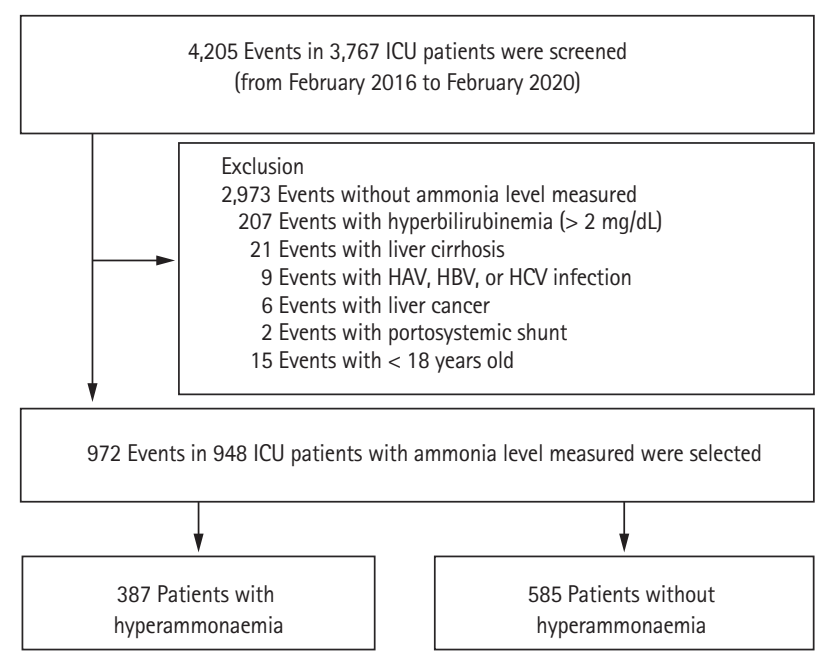

Supplementary Figure 1. Patient recruitment flow chart. ICU, intensive care unit; HAV, hepatitis A virus; HBV, hepatitis $\mathrm{B}$ virus; $\mathrm{HCV}$, hepatitis $\mathrm{C}$ virus. 


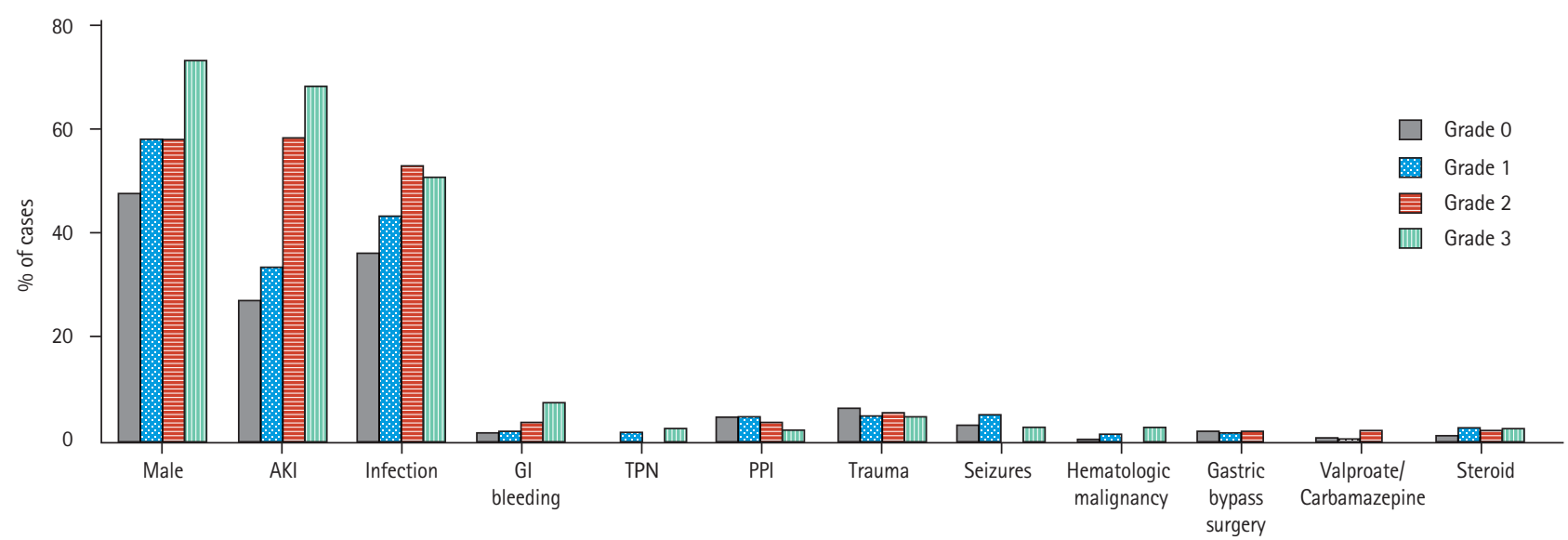

Supplementary Figure 2. Potential factors associated with hyperammonemia in the different ammonia grades $(\mathrm{n}=972)$. AKI, acute kidney injury; GI, gastrointestinal; TPN, total parenteral nutrition; PPI, proton pump inhibitor. 


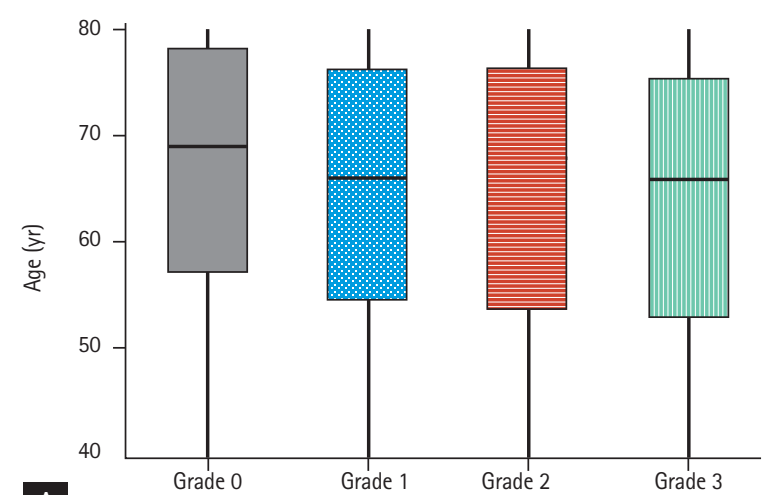

A

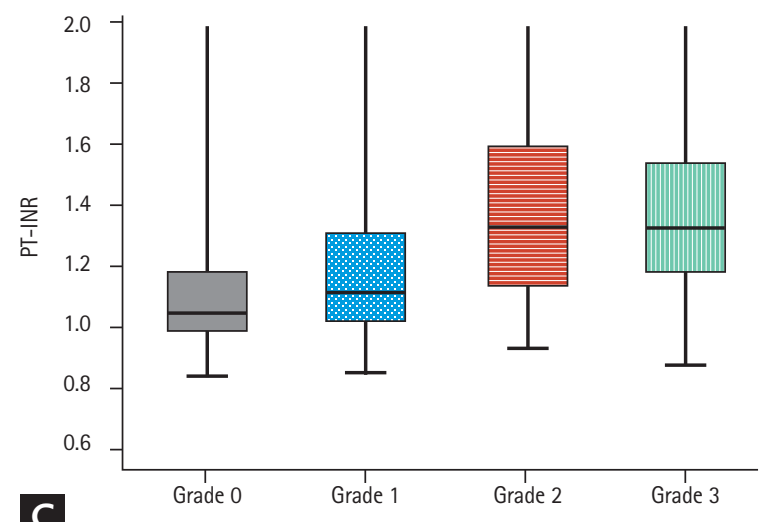

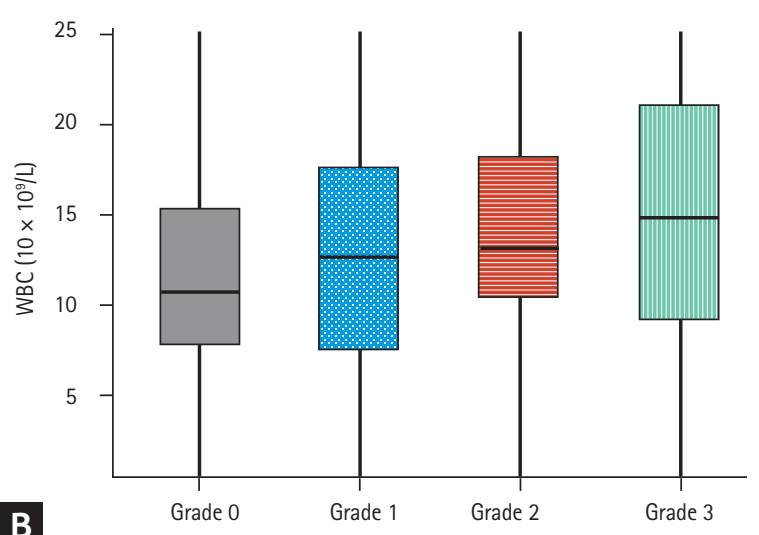

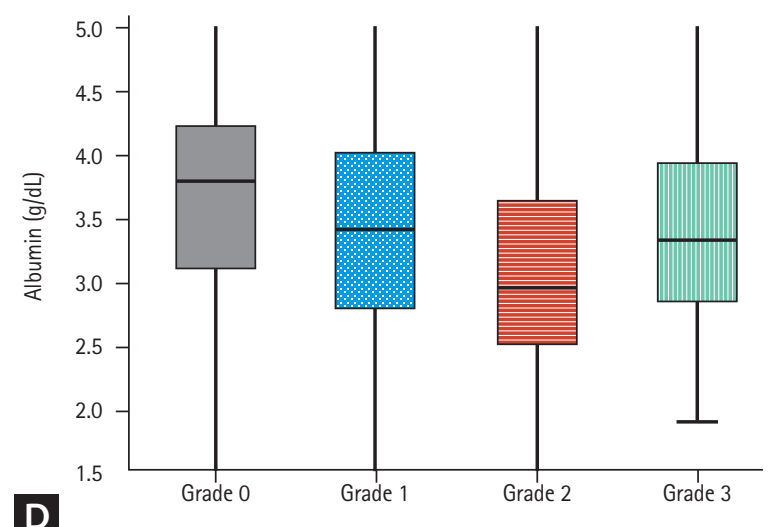

Supplementary Figure 3. Ages and laboratory parameters significantly associated with hyperammonemia in the different ammonia grades $(n=972)$. (A) Age, (B), white blood cell (WBC), (C) prothrombin time-international normalized ratio (PT-INR), and (D) albumin. 